\title{
Projecting the nanoworld: Concepts, results and perspectives of molecular electronics
}

\author{
Giuseppe Maruccio, ${ }^{* a, b}$ Roberto Cingolani $^{a}$ and Ross Rinaldi ${ }^{a}$ \\ ${ }^{a}$ National Nanotechnology Laboratory of INFM, Via per Arnesano, 73100 Lecce, Italy. \\ E-mail: giuseppe.maruccio@unile.it \\ ${ }^{b}$ Department of Physics - University of Lecce, Via per Arnesano, 73100 Lecce, Italy
}

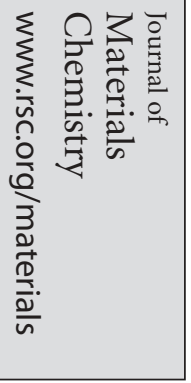

Received 29th September 2003, Accepted 7th November 2003

First published as an Advance Article on the web 13th January 2004

\begin{abstract}
A bottom-up approach is a promising alternative to build nanodevices and/or nanomachines starting from molecular building blocks. The idea of molecular electronics comes from a farsighted paper by Aviram and Ratner, predicting that single molecules with a donor-spacer-acceptor structure would have rectifying properties when placed between two electrodes. Today, molecular electronics is emerging as an alternative to Si-nanoelectronics for building integrated devices. This review aims to give an overview of this emerging field, analysing the concepts, the key results and the perspectives.
\end{abstract}

\section{Introduction-The nanoworld: nanoscience and nanotechnology}

In the last decades, miniaturization has been the key to the major technological innovations and a number of important scientific discoveries. As Richard P. Feynman said in a famous talk to the American Physical Society in 1959: "there is plenty of room at the bottom". Until now the need to make progressively smaller structures has been satisfied by the socalled top-down approach, which exploits the ability to realize smaller and smaller structures using lithography and related techniques ${ }^{1}$ for the construction of both electronic components and microelectromechanical systems (MEMs). Despite the very high resolution reached up to now (Fig. 1), it is current opinion that the actual trend (described by the Moore law in electronics, see Fig. 2) is not further sustainable ${ }^{2}$ using standard (lithographic) approaches, due to intrinsic physical limitations (hundreds of nanometers) and to a remarkable increase in production costs.

A bottom-up approach is a promising alternative to build nanodevices and/or nanomachines starting from molecular building blocks instead of carving lithographically bigger
Giuseppe Maruccio, born on February 1978, graduated in Physics (magna cum laude) in July 2000 at Lecce University with a dissertation on "Quantum Dots Lasers". Since June 2001, he has been a PhD Student at the Physics Department. In 2002, he set up the apparatus for Electrostatic Force Microscopy and Scanning Capacitance Microscopy at NNL. His present research activity includes the investigation of selfassembled layers using scanning probe techniques, the study of electronic and optical properties of mesoscopic and molecular systems/devices and the development of models for nanodevices.

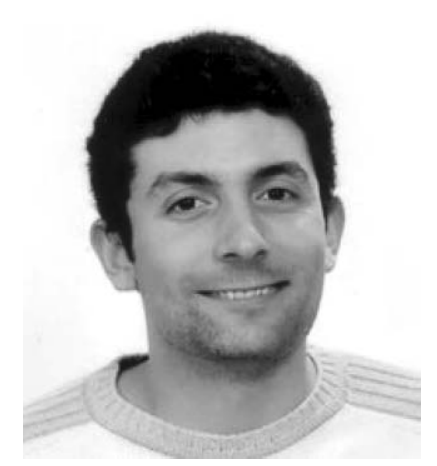

Giuseppe Maruccio

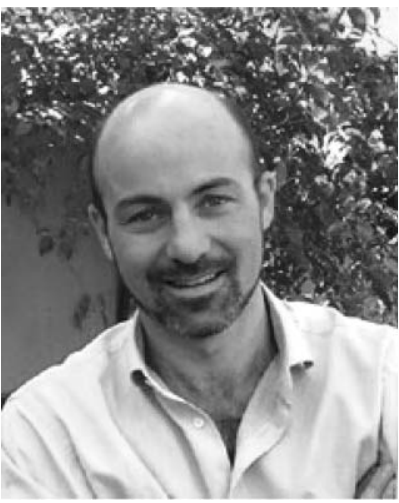

Roberto Cingolani
Roberto Cingolani graduated in Physics in March 1985, got his PhD in Physics at Bari University in Nov. 1988 and the International Certificate of Proficiency in Physics at Scuola Normale Superiore of Pisa in 1990. He was a staff member at Max Planck Institut in Stuttgart (1988-1991), and an invited Professor at Tokyo University in 1997. He held a Faculty Position at Virginia Commonwealth University, Richmond, USA, from 1998. Since 1999 he has been a Full Professor of General Physics at the Engineering faculty in Lecce University. Since 2001 he has been executive Vicepresident of the National Institute for the Physics of Matter, and Director of the
INFM National Nanotechnology Laboratory at Lecce. In 2003 he was appointed President of INFM by the CNR Commisser.

Ross Rinaldi graduated in Physics in July 1991 and got her PhD in 1994. She was appointed lecturer in Condensed Matter Physics in May 1997 and Professor in January 2001. She set up the laboratory of UHV Scanning Tunneling Microscopy at cryogeninc temperatures and a CleanRoom for advanced nanoprocessing of materials. She opened a new research line on nano-biotecnology and nano-bioelectronics and in 2002 she set up a small biology laboratory to handle proteins, DNA and cells.

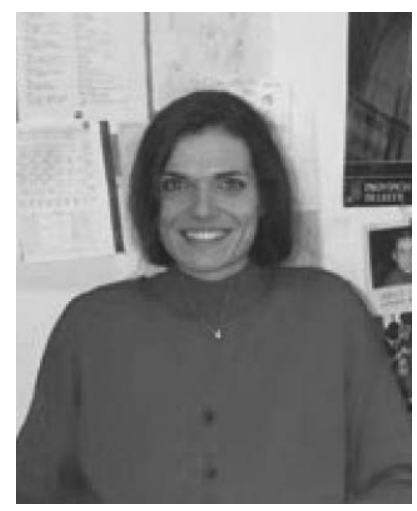

Ross Rinaldi 


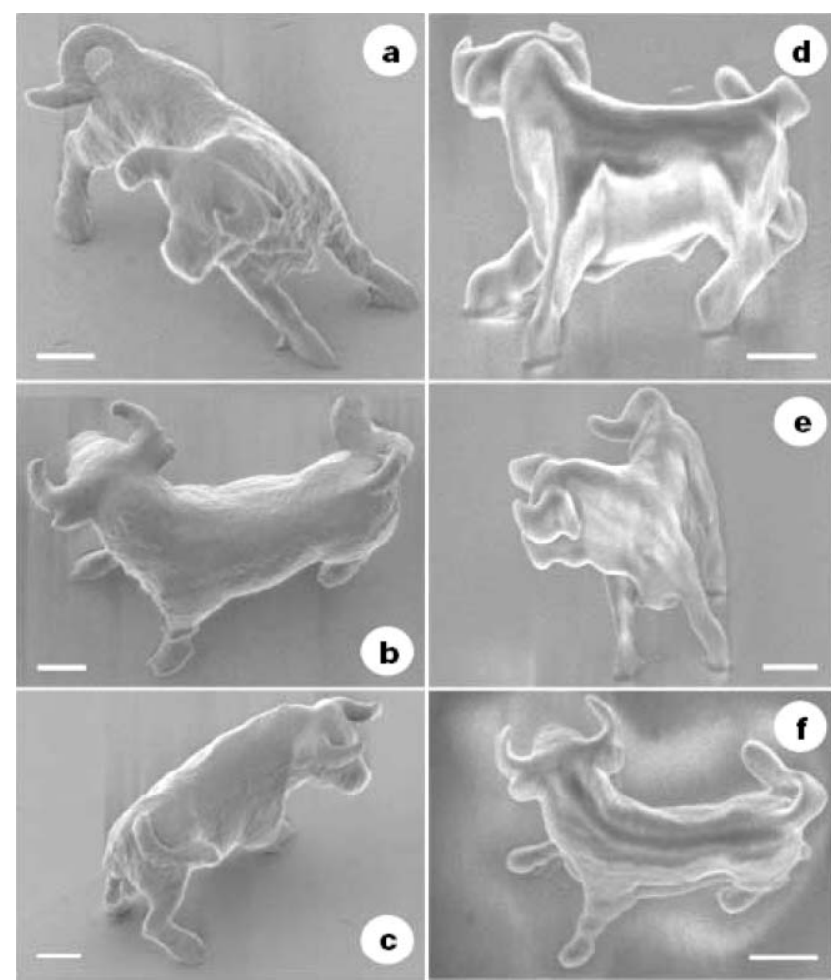

Fig. 1 Fabrication of a bull sculpture $(10 \mu \mathrm{m}$ long and $7 \mu \mathrm{m}$ high, about the size of a red blood cell) produced by raster scanning at subdiffraction-limit resolution using a titanium sapphire laser. Scale bars: $2 \mu \mathrm{m}$. [Reprinted by permission from Kawata et al., Nature, 2001, 412, 697-698. Copyright 2001 Macmillan Publishers Ltd.]

pieces of matter into smaller and smaller chunks. Merging biological, chemical and physical knowledge by means of a cross-disciplinary effort, allow us to extend a number of macroscopic concepts to the nanoworld designing and creating devices and machines that operate via electron and/or nuclear rearrangements. ${ }^{3}$ Recently, key demonstrations of molecularscale devices and machines have been reported, suggesting that the obstacles to the accomplishment of nanotechnology are

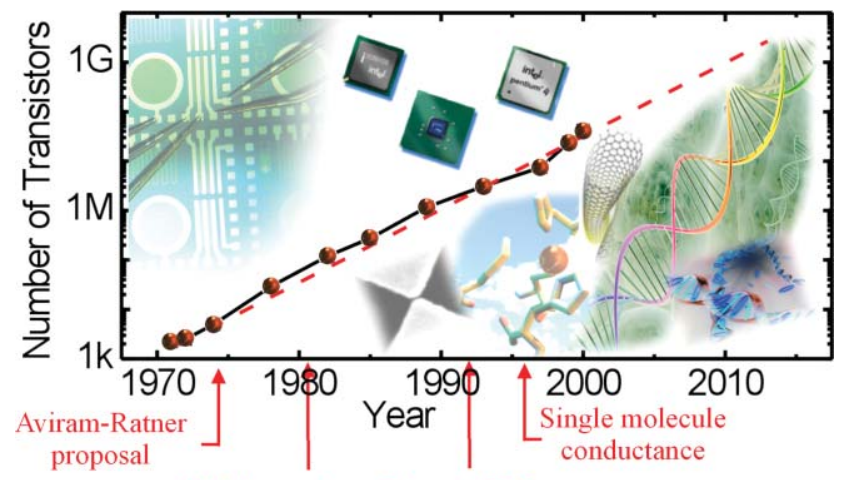

STM Invention Organic FET

Fig. 2 Moore's law, number of transistors as a function of time (points refer to the various processors introduced by Intel: 4004, 8008, 8080, 8086, 286, 386, 486, Pentium, Pentium II, Pentium III and Pentium 4, respectively). In 1965, Gordon Moore, co-founder of Intel, firstly observed and then predicted that the number of transistors per integrated circuit grew exponentially. So far, this prediction has been fulfilled thanks to the continuous advances in miniaturization driven by the progress in lithographic techniques. But now it seems to be in serious danger and modern electronics has to face the restrictions dictated by the laws of physics when the minimum feature-size of a chip approaches $100 \mathrm{~nm}$, at least with current technologies. Thus, gaining the nanometer scale and/or further enhancing the computational capabilities requires a turning point, a change in architecture and the development of conceptually new devices exploiting spin, quantum mechanics and/or molecular building blocks.

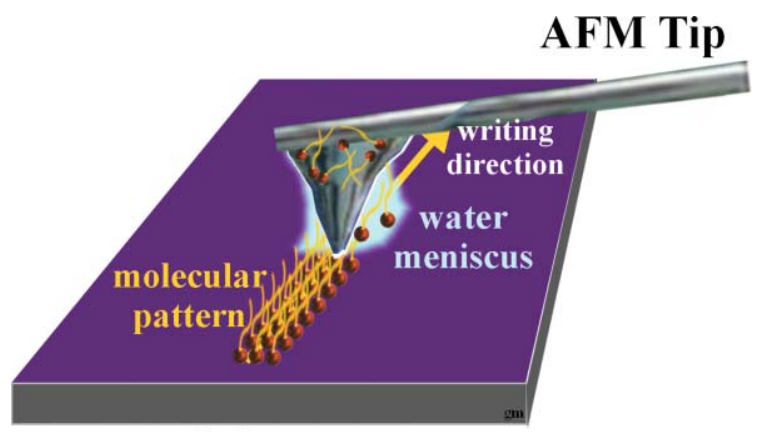

Substrate

Fig. 3 Dip-Pen Nanolithography, ${ }^{33}$ a powerful technique allowing the delivery of molecules to a suitable substrate from a solvent meniscus by directly writing molecular inks from a coated atomic force microscope tip.

more technical than conceptual. In particular, these achievements has been favoured by the advent of new, very powerful techniques capable of seeing and manipulating single molecules, such as scanning probe microscopy and related techniques. For example, Dip-Pen Nanolithography (Fig. 3), developed by Mirkin's group, is a powerful nanopatterning technique which allows one to place molecules on a surface via a solvent meniscus by directly writing molecular inks from a coated atomic force microscope (AFM) tip to a substrate of interest.

Another key factor for the growth of nanoscience and nanotechnology has been the birth of supramolecular chemistry (Fig. 4), namely "a chemistry beyond the molecule, bearing on organized entities of higher complexity that result from the association of two or more chemical species held together by intermolecular forces" as the Lehn definition ${ }^{4}$ states. Chemistry plays a central role in the molecular approach by providing molecular building blocks with thermodynamically driven self-assembly and self-recognition properties. ${ }^{5}$ This issue represents a bridge to biology since self-assembly and selfrecognition are dominant processes in the chemistry of living biological systems. ${ }^{3}$ Moreover, billions of years of evolution have optimised a large variety of biomolecules capable of performing efficiently a lot of different functions. As a consequence, researchers can now look at biology not only to understand it, but also to be inspired in building devices which mimic nature in sensing, ${ }^{6}$ computing, ${ }^{7}$ processing

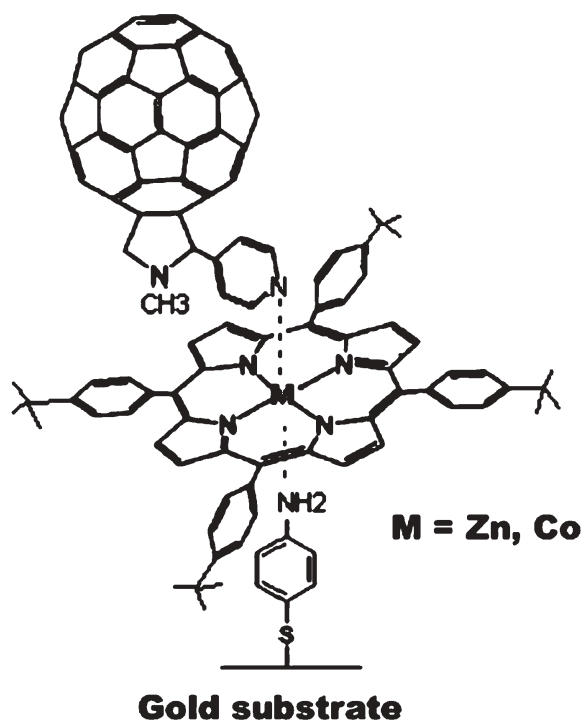

Fig. 4 Supramolecular dyad, involving a heterofullerene acceptor and a metal porphyrin donor, assembled via axial coordination and linked to a gold substrate by means of 4-aminothiophenol. 
signals, storing information and producing energy ${ }^{8}$ as well in creating multifunctional systems and molecular scale motors, ${ }^{9}$ capable of performing mechanical movements. Similarly, several new ideas of novel functional applications have been proposed, such as nanosystems working as molecular fuel tanks for hydrogen storage ${ }^{10}$ flow sensors, ${ }^{11}$ femtogram mass detectors, ${ }^{12}$ AFM probes, ${ }^{13}$ etc.

Nanotechnology is rapidly growing under the stimulus of nanoscience, though a deep understanding of the special rules that control nanosystems is necessary together with the development of the skill needed to manipulate objects on the nanometer scale and to integrate them into working devices.

The objective of this feature article is to give an overview of the emerging field of molecular electronics, analysing the concepts, the key results and the perspectives of the so-called bottom-up approach. In section 2, the concepts and the milestones of molecular electronics are described. In section 3, we report how to deliver and interconnect molecules in order to probe molecular conduction. The key results and the new directions in molecular electronics are then discussed in section 4, giving a description of the state of the art in this field. Finally, we will draw some conclusion in section 5. Due to the rapid growth in this field, this review cannot be considered as exhaustive. Instead, it should be considered as a support for researchers approaching molecular electronics.

\section{The concept and the milestones of molecular electronics}

Since the early 1970s, with the introduction of a $1 \mathrm{~kb}$ DRAM and a $0.75 \mathrm{MHz}$ microprocessor, electronics has been essentially identified with the metal-oxide-semiconductor (MOS) large-scale-integration circuits. ${ }^{14}$ During the past decades, the semiconductor industry has followed Moore's law by the downsizing of the components, such as MOSFET, and the transistor density on integrated circuits has been almost doubled every year, continuously increasing the speed and performance of devices (Fig. 2). The information technology (IT) revolution is based on this exponential growth rate. Even though Moore's law has been valid for three decades, modern electronics has to face the restrictions dictated by the laws of physics when the minimum feature-size of a chip is reduced below $100 \mathrm{~nm}$.

Until now, miniaturization in electronics has been achieved by the so-called top-down approach thanks to the improvement of the lithographic step. However, the current trend is not further sustainable using this strategy due to three fundamental limits. (1) The energy needed to write a bit must be bigger than the average energy of the thermal fluctuations $(k T)$ to avoid bit errors (thermal limit). (2) The energy necessary to read or write a bit and the frequency of the circuits are limited by the uncertainty principle (quantum limit). Finally, (3) a maximum tolerable power density exists (power dissipation limit). Technological and economic limitations related to the growing difficulties in fabricating smaller and smaller devices complete this picture. Gaining the nanometer scale and/or further enhancing computational capabilities requires a turning point, a change in architecture and the development of conceptually new devices ${ }^{7,15,16}$ to overcome the physical and technological limitations of the top-down approach in semiconductor electronic devices fabrication and to satisfy future requirements.

The idea of molecular electronics comes from a farsighted paper by Aviram and Ratner, ${ }^{17}$ predicting that single molecules with a donor-spacer-acceptor structure would have rectifying properties when placed between two electrodes. Today, molecular electronics is emerging as an alternative to Sinanoelectronics for building integrated devices. Though it needs a cross-disciplinary effort (merging chemical, biological

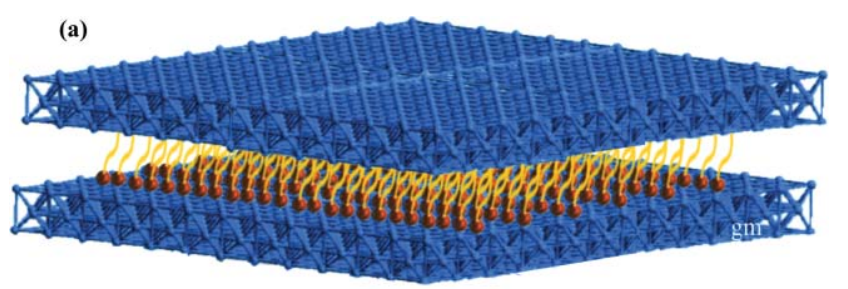

(b)

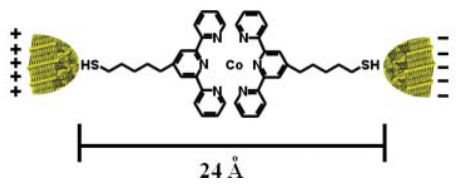

Fig. 5 While applications of molecular materials (a) are numerous, ranging from liquid crystals in displays to dye lasers, light-emitting diodes and plastic transistors, molecular scale electronics (b) have a very promising future starting from the already reported number of working devices based on few or single molecules.

and physical expertise), the molecular approach offers the important advantages of the high reproducibility and small size of the molecular building blocks (naturally identical and with well defined sizes and electronic levels) along with their thermodynamically driven self-assembly and the self-recognition properties. ${ }^{5}$ Molecular materials are already largely used in electronics ${ }^{18}$ and find numerous applications as liquid crystals in displays, dye lasers, light-emitting $\operatorname{diodes}^{19}$ and plastic transistors. ${ }^{20}$ But the development of molecular-scale electronics consisting of molecular electronic devices based on few or single molecules (Fig. 5) is still a challenging direction to future electronics either to produce standard devices (such as diodes and transistors) or to develop new functionalities.

The advances in the synthesis of electronic molecules ${ }^{21}$ with electron donor and acceptor groups and in the fabrication of metallic electrodes with nanometer-scale separation ${ }^{22,23}$ are key elements of the significant progress in this field. Recent results demonstrate that the concept of hybrid molecular devices is useful and practicable for individual components, but a great challenge remains to move from prototypes to commercial devices, because of the intrinsic difficulty to interconnect molecules, to develop fully-reproducible and high-throughput fabrication processes and to combine them in real devices. Moreover, the development of new, powerful techniques to assemble molecular circuits at the nanometer scale is a key requirement for the success of molecular electronics. The conservative architecture that uses metallic wires to interconnect different devices and to form complex circuits is constrained by physical limitations, such as the failure of electrodes by electro-migration. The ideal alternative will be to use molecular wires in order to switch from hybrid molecular devices to an all-molecular electronics, ${ }^{24}$ where the wires and the devices are no longer distinguishable as different components of the network. A digital computer logic at the molecular level (embracing AND, OR and XOR logic gates as well as molecular electronic half and full adders) has been proposed, based on operating molecular devices. But the design of such architecture needs great attention due to the inadequacy of Ohm's and Kirchhoff's laws to describe these circuits, since the addition of a new branch in the network changes the electronic wavefunctions originating new molecules.

In conclusion, molecular electronics has the potential to be the next paradigm for future electronic devices. The main milestones for a development road-map should be the following:

1) two terminal conductance measurements on single molecules;

2) demonstration of two-terminal molecular devices;

3) demonstration of three-terminal molecular devices;

4) integration of functions at the molecular level; 
5) achievement of long-term stability and the development of high-throughput fabrication processes.

\section{Probing, interconnecting and delivering molecules}

\subsection{Probing and interconnecting molecules}

The development of reliable methods to deliver, interconnect and probe molecules is a key issue in molecular electronics as well as in all nanotechnology, with the main difficulty represented by their typical dimensions. To date, various approaches to measure molecular conductivity have been proposed, including scanning probe techniques (both scanning tunneling microscopy ${ }^{25}$ and conductive probe atomic force microscopy ${ }^{26}$ ), mercury drop electrodes, ${ }^{27}$ electrochemical measurements, nanopores, ${ }^{28}$ break junctions ${ }^{29}$ and electrodeposited electrodes.

Two terminal conductance measurements on single molecules have been firstly achieved by scanning probe techniques. Scanning tunneling microscopy (STM) and scanning tunneling spectroscopy (STS $)^{25}$ enable controlled two-terminal measurements and have been widely used to achieve molecular scale resolution imaging, to measure the tunneling current across molecules on conductive substrates (typically gold) and to map the electronic density of states. In ref. 25, S. Datta and coworkers probed self-assembled monolayers of $\alpha, \alpha^{\prime}$-xylyl dithiol molecules on a gold substrate using a scanning tunneling microscope. Good quantitative agreement between the theoretical and experimental current-voltage characteristics was obtained with the tip-molecule distance as the only fitting parameter. The advantage of a scanning probe technique is the possibility to perform simultaneously both topographic nanoscale imaging and electrical transport measurements on material surfaces, and to correlate electrical properties to specific topographic features. However, since the position of the tip and the conductivity of the sample are coupled, it is not easy to extrapolate the conductivity of molecules from STM data because it is difficult to establish exactly the location of the tip with respect to the sample. Conductive probe atomic force microscopy (CP-AFM ${ }^{26}$ circumvents this difficulty since the force feedback allows one to control the position of a metalcoated tip with respect to the substrate, thus decoupling the probe positioning from the conductivity of the sample. Moreover, CP-AFM can be employed to study electrical transport properties on samples which are too resistive to be probed by scanning tunneling microscopy. For example, T. W. Kelley et al. ${ }^{26}$ used CP-AFM to perform point-contact current-voltage measurements on very thin molecular crystals. The drawback of CP-AFM is the need to apply a force, which can perturb the system under investigation and influence its conduction properties, mainly in the case of soft molecular layers such as protein or self-assembled monolayers (SAM). In order to avoid this limitation, R. E. Holmlin et al. ${ }^{27}$ used a drop of $\mathrm{Hg}$ as electrode in metal-insulator-metal junctions to simultaneously achieve electrical signal and minimize the possibility of shorting and mechanically damage the SAM, due to its ability to conform to the sample surface.

A major challenge in molecular electronics is how to interconnect molecules and probe molecular conductivity in real devices working at the nanoscale (both two- and threeterminal devices). This issue requires the fabrication of nanometer spaced electrodes. Since optical lithography suffers from physical limitations (mainly related to diffraction effects), new techniques are needed for patterning below $100 \mathrm{~nm}$. Even though none of the proposed methods equals the advantages of photolithography for low cost and high throughput, a number of lithographic solutions (Extreme Ultraviolet lithography, ${ }^{30}$ X-ray proximity lithography, electron- and ion-beam lithography) are available, and new techniques have been developed to produce very close electrodes. For instance, M. Reed and coworkers $^{29}$ have investigated charge transport through molecules of benzene-1,4-dithiol self-assembled onto the two facing gold electrodes of a mechanically controllable break junction fabricated by the gentle fracture of an electrode by means of mechanical deformation. Another possibility is to constrain molecules in free-standing junctions, which consist of suspended electrodes used to electrostatically trap molecules and/or nanoparticles by monitoring the current and interrupting the trapping fields at the first increase in conductivity. Among these various alternatives, planar nanojunctionsconsisting of two facing metallic electrodes separated by an insulating medium and fabricated by electron beam lithography (EBL) and lift-off processes-are ideal to implement molecular devices since they have a high process yield (typically around $90 \%$ of the nanojunctions are good with an opencircuit resistance as high as $100-200 \mathrm{G} \Omega^{31}$ ) and provide the opportunity to perform transport experiments on molecules and/or nanostructures self-assembled at the surface of a solid, as well as to fabricate field effect devices by adding a third electrode at the bottom of the device. Recently, Y. V. Kervennic et $a .^{23}$ succeeded in producing pairs of platinum electrodes with separation down to $3.5 \mathrm{~nm}$ combining electron beam lithography (EBL) and chemical electrodeposition. The separation between EBL-fabricated electrodes is reduced to atomic separation by monitoring the interelectrode conductance and stopping the electrodeposition process at predefined conductance values.

\subsection{Delivering molecules}

Molecular scale assembly of individual components in working devices is another challenge of molecular electronics. In the last few years, great attention has been focused on the development of techniques capable of fabricating molecular layers and patterns (evaporation, spin-coating, Langmuir-Blodgett techniques, thermodynamically-driven self-assembly and soft lithography) or to deliver molecules to specific locations (electrostatic trapping, dip pen and related techniques, AFM and membrane nanostencil, optical tweezers) (Fig. 6). All these fabrication strategies have their own advantages and disadvantages. Spin-coating is fast and cheap but films are strongly anisotropic and inhomogeneous. On the contrary, thermodynamically-driven self-assembly opens the way to the fabrication of engineered highly ordered layers, taking advantage of the specific reactivity of molecules having functional groups with affinity for specific surface atoms and/or molecular sites. However, detailed knowledge of the positions, identities and affinity of the functional groups is required in order to develop a reasonable linkage strategy.

Transport characterization techniques

\begin{tabular}{|c|c|c|c|c|c|}
\hline Two-terminal measurements & \multicolumn{2}{|c|}{ STM } & \multicolumn{2}{|l|}{ CP-AFM } & $\begin{array}{l}\text { Mercury drop } \\
\text { electrodes }\end{array}$ \\
\hline Junctions for real devices & \multicolumn{2}{|c|}{ Break junctions } & \multicolumn{2}{|c|}{ Free standing junctions } & Planar junctions \\
\hline \multicolumn{6}{|c|}{ Nanopatterning and Molecular Delivering Methods } \\
\hline \multicolumn{2}{|l|}{ Local/Scanning } & \multicolumn{2}{|c|}{ Static/Large Area } & \multicolumn{2}{|r|}{ Type } \\
\hline \multicolumn{2}{|l|}{ Optical tweezers } & \multicolumn{2}{|c|}{$\begin{array}{l}\text { Extreme UV and X-Ray } \\
\text { proximity Lithography }\end{array}$} & \multicolumn{2}{|c|}{ Light-based } \\
\hline \multicolumn{2}{|c|}{$\begin{array}{l}\text { Electrostatic trapping; } \\
\text { electron- and ion- beam } \\
\text { lithography }\end{array}$} & & & \multicolumn{2}{|c|}{ Electromagnetic } \\
\hline \multicolumn{2}{|c|}{$\begin{array}{l}\text { AFM Nanolithography, } \\
\text { AFM storage, "Millipede" }\end{array}$} & \multicolumn{2}{|c|}{ Nanoimprint lithography } & \multicolumn{2}{|c|}{ Thermo-mechanical } \\
\hline \multicolumn{2}{|l|}{$\begin{array}{l}\text { AFM nanostencil, } \\
\text { Membranes Nanostencil }\end{array}$} & \multicolumn{2}{|c|}{$\begin{array}{l}\text { Evaporation, spin- } \\
\text { coating, Langmuir- } \\
\text { Blodgett technique }\end{array}$} & \multicolumn{2}{|l|}{ Deposition } \\
\hline \multicolumn{2}{|l|}{$\begin{array}{l}\text { Dip Pen and Parallel Dip } \\
\text { Pen Nanolithography }\end{array}$} & \multicolumn{2}{|c|}{ Soft Lithography } & \multicolumn{2}{|c|}{ Molecular Delivery } \\
\hline \multicolumn{2}{|l|}{ Molecular Lithography } & \multicolumn{2}{|c|}{$\begin{array}{l}\text { Self-assembled } \\
\text { monolayers }\end{array}$} & \multicolumn{2}{|c|}{$\begin{array}{l}\text { Self-assembly/self } \\
\text { recognition }\end{array}$} \\
\hline
\end{tabular}

Fig. 6 Transport characterization techniques and nanopatterning and molecular delivering methods. 
Finally, soft lithography consists of different fabrication techniques (embracing imprint and stamping methods) which allow us to create patterns on the surface of a material using a pattern-transfer element - a rigid stamp - made of an elastomer, a thermoplastic polymer network that deforms under an applied force and recovers its shape when the force is released. Nanoimprint lithography ${ }^{32}$ is a low coast, high throughput technique which provides high resolution features and a large processing area but it does not allow control at the molecular scale.

In addition to these techniques - parallel and static - a number of scanning methods - serial or parallel-have been developed to place molecules in specific, well-established locations (for examples in the gap between two nanoelectrodes). Dip-Pen Nanolithography (DPN), proposed by Mirkin's group, ${ }^{33}$ is a powerful resistless nanopatterning technique allowing the delivery of molecules to a suitable substrate from a solvent meniscus. The concept of dip-pen nanolithography is quite simple. A conventional silicon nitride AFM tip literally draws molecules of virtually any material from the solvent meniscus to a surface of interest ("paper"). The molecular "ink" consists of a chemically reactive material placed on the tip. The fine controls of the tip position and the chemisorption of ink molecules as the tip is scanned across the substrate enable the deposition of organic molecules in a nanometer scale region. Using DPN it is currently possible to achieve high-resolution patterns (linewidth as small as $15 \mathrm{~nm}$ and less than $10 \mathrm{~nm}$ spatial resolution). The main parameters affecting the nanopatterning results are the diffusion and the relative humidity. When deposited onto solid surfaces, molecules diffuse out, therefore dramatically influencing the spatial resolution. On the other hand, the relative humidity controls the dimension of the naturally-formed water droplet between the substrate and the AFM tip and thus the effective contact area. Another limiting parameter is the tip radius of curvature. Due to the high resolution and the possibility to use the same apparatus to image and to write a pattern, DPN is a very powerful tool for molecular electronics. More recently, Mirkin's group has also developed a eight-pen nanoplotter capable of doing parallel DPN ${ }^{34}$ using a dense array of AFM tips, and has employed DPN to generate DNA-based templates capable of guiding the assembly of nanoscale building blocks. ${ }^{35}$ This technique opens the way to placing molecules and/or nanostructures at established positions. A different approach enables M. Cavallini et al. ${ }^{36}$ to store information by writing with a scanning probe on thin films of rotaxanes. A load force above the $2 \mathrm{nN}$ threshold results in the formation of localized and regularly spaced dots along the scanning direction, due to the structure of rotaxanes which consist of two mechanically interlocked compounds. The number, size and spacing of dots can be controlled in order to write a specified supramolecular surface pattern. Another interesting tool for the generation of nanometer patterns is the "nanostencil", a new resistless lithographic technique based on a high resolution scanning shadow mask. Complex patterns can be realized by local physical vapour deposition through one or more apertures in a cantilever or membrane exposing a movable sample to a collimated atomic or molecular beam in high or ultra-high vacuum environment (which allows the fabrication of atomically clean devices). Recently M. Kölbel et $a l^{37}$ proposed a monolayer-modified nanostencil to solve the problem of the gradual clogging of the aperture. Finally, light can also be used to move matter and manipulate nanoscopic objects, because photons carry momentum. Optical tweezers are built on this principle. Nanoparticles are trapped in the focus of a laser beam and serially moved. The force felt by the trapped particle is primarily given by the dipole and by the gradient force due to the interaction of a dielectric object with light and lies in the $\mathrm{pN}$ range, therefore allowing the manipulation of very fragile structures. Recently, MacDonald et al. have constructed threedimensional trapped structures within an optical tweezers setup using an interferometric pattern between two laser beams. These results point towards the creation of extended 3D crystalline structures. ${ }^{38}$ Assembling functional molecular devices with this serial manipulation approach is, however, extremely slow and thus technologically unattractive and not suitable for mass production. An alternative, promising strategy to produce large-scale functional circuits at the molecular level is so-called "molecular lithography" exploiting self-recognition and self-assembly of molecules on surfaces (a parallel process). The sequence-specific molecular lithography of Keren et al. ${ }^{39}$ is particularly promising. The information encoded in the DNA molecules replaces the masks used in conventional lithography, while a RecA protein serves as the resist. This technique enables high resolution, the fabrication of three-way junctions (branchpoints) and the sequence-specific positioning of molecular objects. Besides proteins, genetically engineered viruses have also been employed to order nanostructures. ${ }^{40}$

\section{Key results and new directions in molecular electronics}

\subsection{Two-terminal devices}

Starting from the proposal of Aviram and Ratner, ${ }^{17}$ molecular rectification was first observed in monolayers sandwiched between two metallic electrodes and produced using Langmuir-Blodgett or self-assembled techniques. In this case, a macroscopic number of molecules with a donor-spaceracceptor structure is involved in conduction. In the AviramRatner ansatz (Fig. 7), a D- $\sigma-\mathrm{A}$ molecule causes rectification because the excited zwitterion $\mathrm{D}^{+}-\sigma-\mathrm{A}^{-}$is relatively accessible from the ground neutral state $\mathrm{D}-\sigma-\mathrm{A}$, while the opposite zwitterionic state $\mathrm{D}^{-}-\sigma-\mathrm{A}^{+}$lies several $\mathrm{eV}$ higher in energy. Thus, under forward bias, electrons can be transferred between the metallic leads $M_{1}$ and $M_{2}$ in two steps. First, electrons flow from the cathode to the acceptor and from the donor to the anode by elastic tunneling (ET) involving the two electrodes. Afterwards, an intervalence transfer (IVT) occurs in the molecule, which recovers the ground state. The net result is that electrons flow from the cathode to the anode:

$$
\begin{gathered}
\mathrm{M}_{1}|\mathrm{D}-\sigma-\mathrm{A}| \mathrm{M}_{2} \stackrel{\mathrm{ET}}{\longrightarrow} \mathrm{M}_{1}^{-}\left|\mathrm{D}^{+}-\sigma-\mathrm{A}^{-}\right| \mathrm{M}_{2}^{+} \stackrel{\text { IVT }}{\longrightarrow} \\
\mathrm{M}_{1}^{-}|\mathrm{D}-\sigma-\mathrm{A}| \mathrm{M}_{2}^{+}
\end{gathered}
$$

A similar process under reverse bias, $\mathrm{M}_{1}|\mathrm{D}-\sigma-\mathrm{A}| \mathrm{M}_{2} \stackrel{\mathrm{ET}}{\longrightarrow} \mathrm{M}_{1}^{+}\left|\mathrm{D}^{-}-\sigma-\mathrm{A}^{+}\right| \mathrm{M}_{2}^{-}, \quad$ is quenched since donors are bad acceptors, and acceptors are bad donors. If the competing reverse bias mechanism (autoionization

\section{AVIRAM-RATNER MODEL}

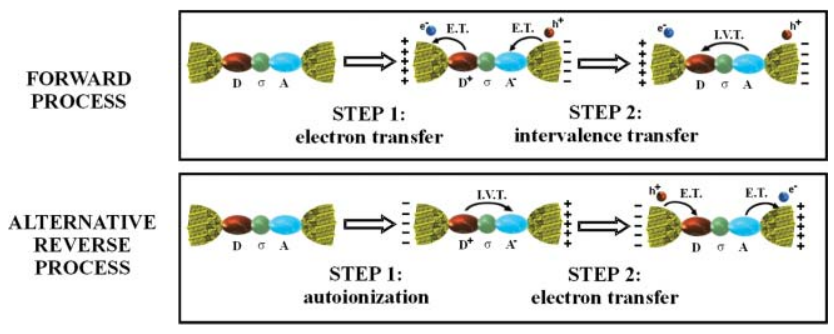

Fig. 7 Schematic picture of the Aviram-Ratner electron-transfer mechanism involving a $\mathrm{D}-\sigma-\mathrm{A}$ molecules and two metallic electrodes. In the forward process, at first electrons move from the cathode to the acceptor and from the donor to the anode. Then, molecules recover the ground state by means of an intervalence transfer. To have rectification, the reverse bias process (involving autoionization) must be infrequent. 


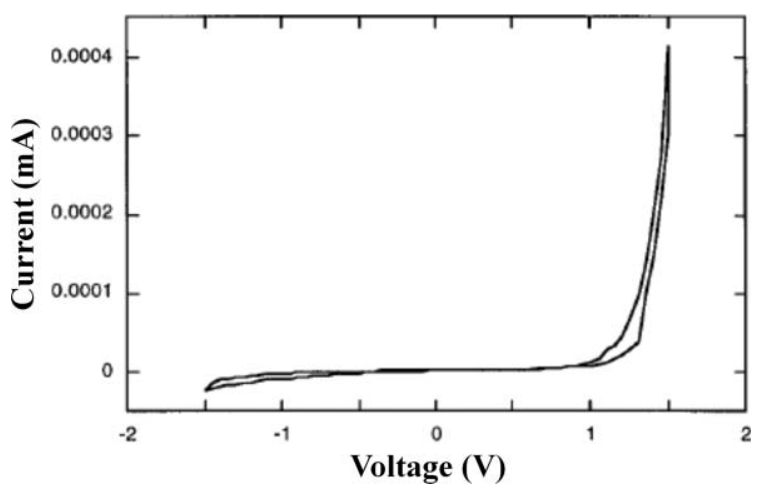

Fig. 8 Current-voltage characteristic of the device reported by Metzger et $\mathrm{al}^{41}$ Transport through a hexadecylquinolinium tricyanoquinodimethanide monolayer exhibits a very high rectification (rectification ratio around 26). [Reprinted with permission from Metzger et al., J. Am. Chem. Soc., 1997, 119, 10455-10466, where further experimental data are given. Copyright 1997 American Chemical Society]

followed by charge migration to the two metal electrodes) is unlikely, molecular rectification occurs. Metzger and coworkers ${ }^{41}$ provided experimental proof for the validity of the Aviram-Ratner design principle also in the case of a ground-state zwitterions $\mathrm{D}^{+}-\pi-\mathrm{A}^{-}$with the neutral form as excited state. They observed rectification ratios $\mathrm{R} R=I(V) / I(-V)$ up to 26 in Langmuir-Blodgett multilayers and monolayers of hexadecylquinolinium tricyanoquinodimethanide (Fig. 8).

The first transport experiments on few or individual molecules were made possible by the scanning tunneling microscope. But in the last decade, for the first time the length of synthesized molecular wires and the inter-electrode distance in nanofabricated junctions became comparable, enabling one to interconnect single molecules in real devices (e.g. in the case of mechanically controllable break junctions or nanoelectrodes fabricated by means of electron beam lithography ${ }^{22}$ and/or electrodeposition techniques ${ }^{23}$ ). When using two metallic electrodes, the main issues are how to deliver molecules and how to avoid the failure of nanojunctions due to the formation of electrical shorts during operation.

In 1999, J. Chen and colleagues ${ }^{28}$ succeeded in probing conduction from a small group of molecules. Molecules containing a nitroamine redox center were self-assembled in a nanopore on a gold surface using sulfur atoms as alligator clips. For the first time in a molecular device, negative differential resistance and large on-off ratios were observed (Fig. 9a). The peak voltage position decreased monotonically at a rate of $9 \mathrm{mV} \mathrm{K}^{-1}$ as the temperature increased. In a following work, J. M. Seminario et al. ${ }^{42}$ explained this electrical
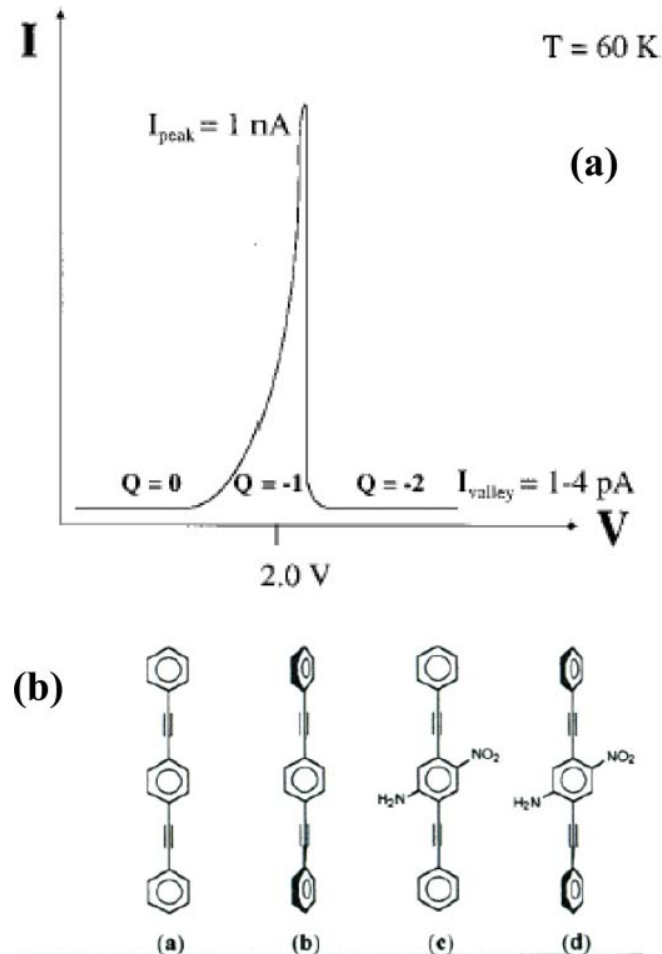
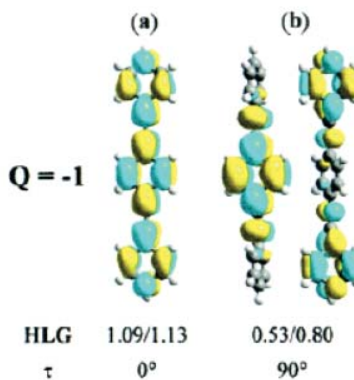

$0.53 / 0.80$

$90^{\circ}$

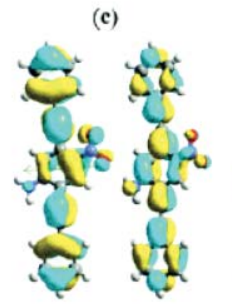

(d)

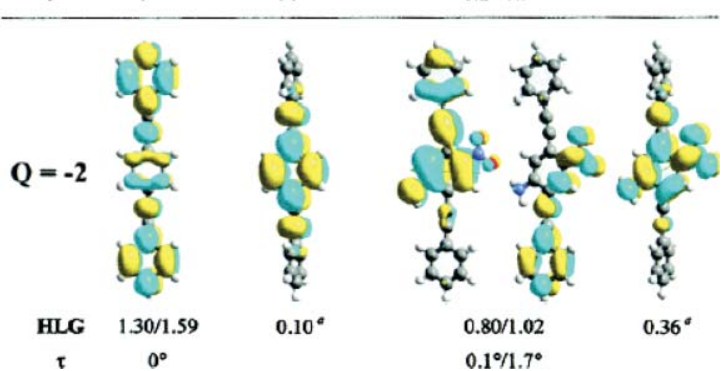

Fig. 9 (a) Characteristics of the molecular resonant tunneling diode realized by Chen and coworkers. ${ }^{28}$ At low voltage the system behaves as an insulator, then current grows up to a maximum before drastically returning to the pA range. The molecular conductivity is determined by the charge $Q$ which causes the resonant tunneling behaviour. (b) LUMO plots, HLG energies (in eV) and torsion angles for the optimised molecular structures. ${ }^{42}$ Density functional calculations performed by J. M. Seminario et al. ${ }^{42}$ explain the experimental results evidencing the existence of an electron transfer pathway which joins both ends of molecules only when $Q=-1$ (conductive state). [Reprinted with permission from Seminario et al., J.Am.Chem.Soc., 2000, 122, 3015-3020. Copyright 2000 American Chemical Society]. See original papers for further details. ${ }^{28,42}$ 


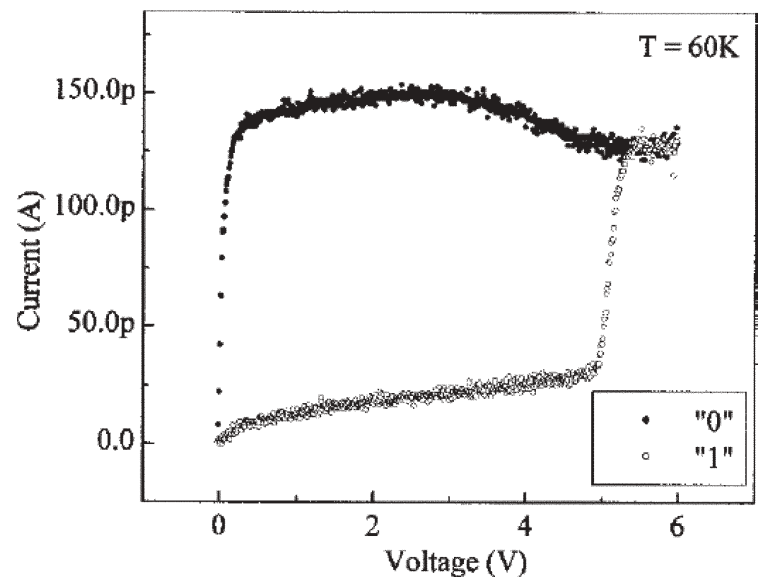

Fig. 10 Electrical characteristics of the molecular random access memory reported by $\mathrm{M}$. Reed and colleagues ${ }^{43}$ at $60 \mathrm{~K}$. The presence of both high and low conductivity states is clear. As a function of time, the current decays exponentially with a time constant (bit retention time) of $789 \mathrm{~s}$ at $260 \mathrm{~K}$. The temperature dependence of the bit retention time exhibits an activation energy around $75 \mathrm{meV}$. [Reprinted with permission from Reed et al., Appl. Phys. Lett., 2001, 78, 3735-3737. Copyright 2001 American Institute of Physics]

characteristic by density functional theory calculations. The resonant tunneling diode (RTD) behaviour is due to a modification of the electronic wavefunctions extending through the molecules. The critical parameters are the HOMO-LUMO gap (HLG) and the spatial extents of the unoccupied orbitals, since delocalisation is needed to have electron transport. Their results showed how the existence of electronic pathways in the molecule depends strongly on the charge $Q$ (Fig. 9b) which enables and disables charge transport upon bias. When $Q=0$, there is no connection of the LUMO between the two terminals of the molecules and no conduction channels exist. However, as the voltage increases, the molecule becomes charged to -1 , the LUMO orbital extends over the two ends of the molecules and an electronic pathways connects the two electrodes, yielding maximum transport. Finally, when $Q=-2$, again the molecular LUMO does not connect both ends and transport is quenched. M. A. Reed and coworkers ${ }^{43}$ also succeeded in the fabrication of a molecular random access memory cell for the programmable storage of digital information, in the form of charges in two conductivity states. The switch between the low (defined as " 0 ") and high (defined as " 1 ") conductivity states is obtained by applying a voltage pulse that writes the bit of information (Fig. 10). The stored bit is unaffected by the following read pulses. The retention time depends exponentially on $1 / T$, where $T$ is the temperature (Fig. 10).

\subsection{Three-terminal devices}

A crucial step for the development of a molecular electronics roadmap is the demonstration of a three-terminal device (a transistor) consisting of source (s) and drain (d) contacts interconnected by a molecular layer, and a third contact (gate) to modulate the source-drain current $\left(I_{\mathrm{ds}}\right)$. The basic concept is to control the conductance of a molecule by an electric field. A room-temperature transistor based on a single carbon nanotube has been demonstrated by S. J. Tans and colleagues. ${ }^{44}$ Depending on the tube indices $(\mathrm{n}, \mathrm{m}),{ }^{45}$ carbon nanotubes can behave as conductors, semiconductors or insulators. In ref. 44 , as the gate voltage increased, a low-conductive region appeared and developed around $V_{\mathrm{ds}}=0 \mathrm{~V}$, and the conductivity of the device in this region changed by about six orders of magnitude upon a change of $10 \mathrm{~V}$ in the gate voltage, as a consequence of the modulation of the nanotube band structure induced by the gate and drain-source voltages (Fig. 11). The authors explained this behaviour like in a conventional semiconductor

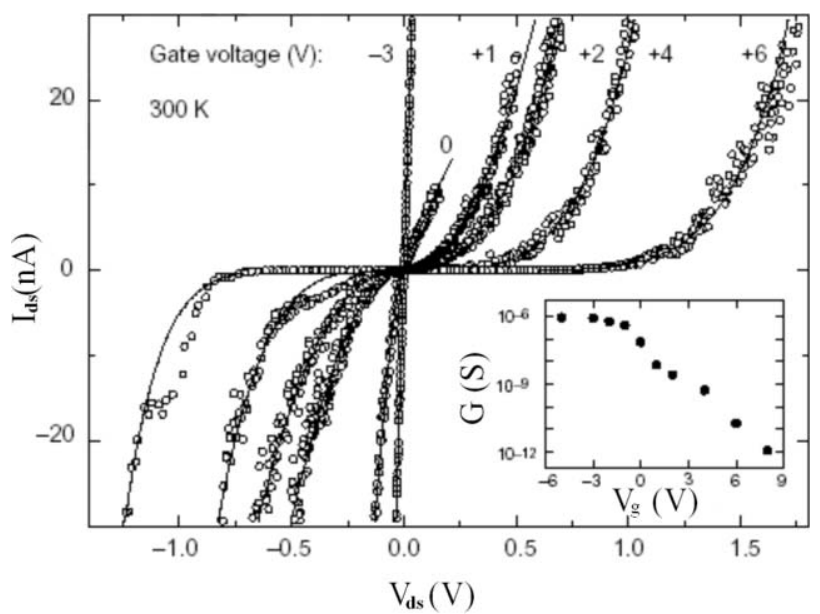

Fig. 11 Characteristics of the room-temperature transistor based on a single carbon nanotube. Dependence of the drain-source current $\left(I_{\mathrm{ds}}\right)$ on the voltage $\left(V_{\mathrm{ds}}\right)$ at discrete gate voltages $\left(V_{\mathrm{g}}\right)$. As the gate voltage increase, a low-current region appears and develops around $V_{\mathrm{ds}}=0 \mathrm{~V}$. Inset. Conductivity at $V_{\mathrm{ds}}=0 \mathrm{~V}$ as a function of the gate voltage. A change of about six order of magnitude is observed in a gate voltage range of $10 \mathrm{~V}$. [Reprinted by permission from Tans et al., Nature, 1998, 393, 49-52 Copyright 1998 Macmillan Publishers Ltd.]

device (the barrier injection transit time (BARITT) diode). The gain of this device was 0.35 . A similar device exhibiting a maximum gain voltage of 0.5 has been reported by J. Appenzeller et al. ${ }^{46} \mathrm{~A}$ single electron transistor working at room temperature and based on carbon nanotubes has also been demonstrated. ${ }^{47}$

Ideally, one can plan to use the central part of a molecule for functions (conduction, rectification, resonant tunneling, switching and storage) while the functional endgroups would provide the links to the electrodes through a self-assembly mechanism. The experiment reported by Park et al. ${ }^{48}$ shows how a short molecule, or even a single atom, can be used to fabricate a working nanodevice. A gap about 1-2 nm wide was fabricated from a wire broken by electromigration at cryogenic temperature. The authors investigated two related molecules containing a Co ion bonded to two terpyridinyl linkers with thiol end groups, which provide linking capability to the two gold electrodes. The signature of a single-electron transistor has been observed (Fig. 12). Conduction was absent up to a threshold voltage which depends on the gate voltage. Then the current rises in steps and the existence of a fine structure of current steps near the voltage threshold was evidenced in the colour plot of the differential conductance. This peculiar behaviour is due to single electron phenomena, related to the charging of the Co ion whose oxidation state was modified by the gate voltage. Current was blocked until an electron gained sufficient energy to tunnel onto the island. A peak in the differential conductance at $V_{\mathrm{ds}}=0$ which splits under magnetic field and exhibits a logarithmic temperature dependence was the signature of the Kondo peak.

\subsection{Biomolecular devices}

Another emerging field of research aims at joining biology and nanotechnology. Using biomolecules as electronic components is an intriguing route to molecular electronics which enables the exploitation of the natural self-assembly and self-recognition properties of these molecules. Biomolecules are particularly promising components, because their functions (binding, catalysis, ion-pumping, sensing and self-assembly) have been optimised during billions of years of evolution. For example, DNA oligonucleotides have unique and predictable recognition capabilities that make them useful for different applications ranging from DNA-computing to molecular lithography. 

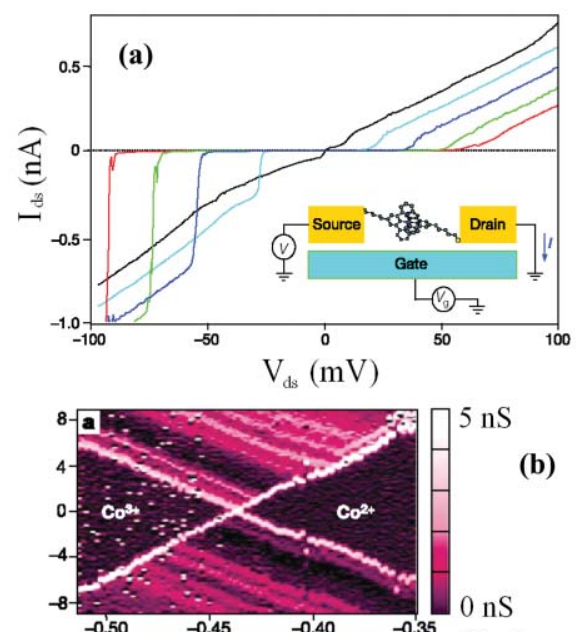

(b)
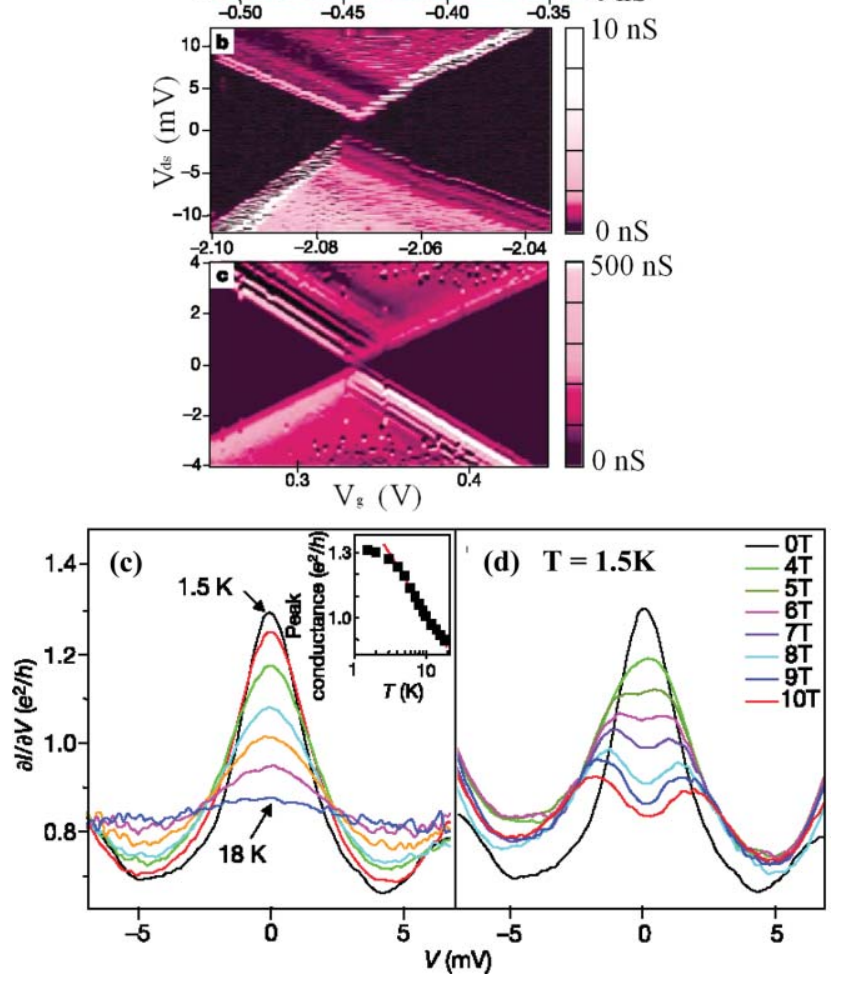

Fig. 12 (a) Schematics and current-voltage characteristics of the single-atom transistor fabricated by Park et al. at different gate voltages from $-0.4 \mathrm{~V}$ (red curve) to $-1.0 \mathrm{~V}$ (black one). (b) Colour map of the differential conductance-numerically extrapolated by differentiating the $I-V$ curves - as a function of both drain-source and gate potentials. (c-d) Temperature and magnetic-field dependences of the Kondo peak, respectively. [Reprinted by permission from Park et al., Nature, 2002, 417, 722-725. Copyright 2002 Macmillan Publishers Ltd.]

Moreover, specific enzymes can be used to process with angstrom-level accuracy nucleic acid (using nucleases and polymerises) or to produce powerful biosensors. ${ }^{6}$

Recently, there has also been a lot of theoretical and experimental work aimed at assessing the problem of the electrical transport properties of DNA by means of fluorescence-quenching and electrical-transport measurements. For example, D. Porath et al. ${ }^{49}$ investigated conduction across individual $10.4 \mathrm{~nm}$ long, double-stranded $\operatorname{poly}(\mathrm{G})-\operatorname{poly}(\mathrm{C})$ DNA molecules electrostatically trapped between two metallic nanoelectrodes. They observed large bandgap semiconductor behaviour and currents in the $\mathrm{nA}$ range $(1 \mathrm{nA}$ at $V=4 \mathrm{~V})$. The low-current region widened as the temperature increased. Two different peaks were present in the differential conductance curves and suggested a molecular-band mediated transport mechanism.

In this context, G. Maruccio et al. employed a single modified DNA base, the lipophilic deoxyguanosine, to fabricate electronic devices. Guanosine was chosen due to it having the lowest oxidation potential among the DNA bases, which favours transport, and its self-assembling properties related to its peculiar sequence of $\mathrm{H}$-bond donor and acceptor groups. They succeeded in the fabrication of a prototype field effect transistor based on this modified DNA base (Fig. 13) with maximum voltage gain of 0.76 , which is higher than the gain reported in similar devices. ${ }^{50}$ Unlike other molecular electronics devices based on carbon nanotubes, this prototype FET is based on ordered and self-assembled layers, instead of a single molecule with tremendous interconnection problems. ${ }^{51}$ The transistor was fabricated by cast deposition of a droplet of the deoxyguanosine derivative in chloroform solution between the source and drain contacts consisting of two EBL-fabricated chromium/gold electrodes separated by a distance of 20 $100 \mathrm{~nm}$, while the control electrode was a layer of silver deposited on the back of the $\mathrm{Si} / \mathrm{SiO}_{2}$ substrate. The guanosinebased FET operated at room temperature and ambient pressure and exhibited a voltage threshold for the conduction which could be modulated by the gate voltage. The electrical characteristics were explained in terms of resonant tunneling, the threshold voltage being defined by the alignment between the molecular minibands and the Fermi level in the electrodes. The shift in the threshold voltage was related to a modification of the molecular minibands induced by the gate voltage.

More recently, G. Maruccio et al. ${ }^{52}$ succeeded in the fabrication of a protein field-effect transistor (Pro-FET) based on the blue copper protein azurin and operating at room temperature and ambient pressure (Fig. 14). The possibility of covalently binding proteins in ordered layers onto $\mathrm{Si} / \mathrm{SiO}_{2}$ substrates and their electron transfer capability involving the redox sites, make azurin interesting for molecular electronic applications. The transfer characteristic of the ProFET exhibited a pronounced resonance due to the switch from n-MOS FET behaviour to p-MOS FET behaviour. Carrier transport through the device was explained in terms of equilibrium between the two possible oxidation states $\mathrm{Cu}(\mathrm{I})$ and $\mathrm{Cu}(\mathrm{II})$ of the redox site, and of the reversible redox reaction $\mathrm{Cu}^{2+}+\mathrm{e}^{-} \rightleftharpoons \mathrm{Cu}^{+}$which reconverts continuously the copper oxidized state $\mathrm{Cu}$ (II) into the reduced state $\mathrm{Cu}(\mathrm{I})$ in adjacent proteins. In future, the enzymatic redox partners of azurin like the nitrate, nitrite and nitric oxide reductases could be used in combination with the immobilised blue copper proteins to fabricate powerful biosensors.

\subsection{Need for molecular electronics}

To transfer molecular electronic devices to mass production at the industrial level, device ageing, lifetime and reproducibility are the major challenges and difficulties. The need for high throughput and long-term stability remains a critical issue for practical applications and is among the main obstacles to the accomplishment of reliable hybrid molecular electronics. The sensitivity of conduction to the molecular arrangement and to the organic-inorganic interface properties is still too high and limits device reproducibility. In particular, the strong dependence of the transport properties on the self-assembly stepdue to local changes in the organization of molecules - could be reduced using covalently-bonded molecular layers by means of thermodynamically driven processes, which exploit different functional groups (self-assembled systems based on weak interactions are too influenced by external perturbations such as a change of solvent, $\mathrm{pH}$, etc.). Moreover, degradation during operation is a serious drawback intrinsic to molecular devices, as widely reported in the literature [see for example refs. 41, 48, 53-55]. As a number of recent experiments and devices demonstrated, problems are more technical than conceptual and after the demonstration of working devices, the achievement of device reproducibility and high stability in time 


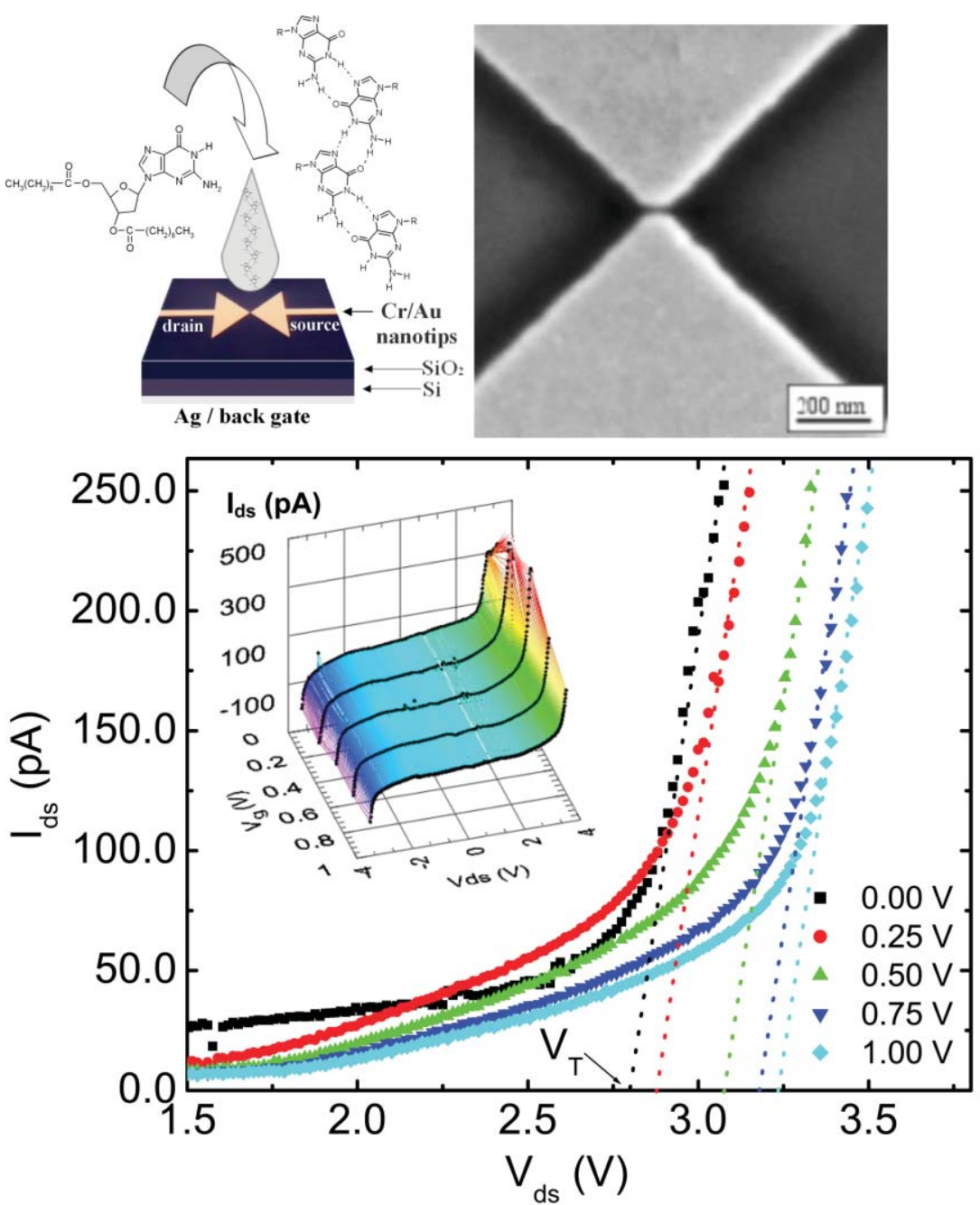

Fig. 13 (Top left) Self assembly and cast deposition of $\mathrm{dG}\left(\mathrm{C}_{10}\right)_{2}$ on the three-terminal device, consisting of two arrow-shaped $\mathrm{Cr} / \mathrm{Au}$ electrodes on a $\mathrm{SiO}_{2}$ substrate and a third $\mathrm{Ag}$ back electrode (not to scale). (Top right) High magnification SEM image of two Cr/Au nanotips with separation of $20 \mathrm{~nm}$. (Bottom) Characteristics of the FET at different gate voltages $\left(V_{\mathrm{G}}\right)$. The dashed lines extrapolate the voltage threshold $V_{\mathrm{T}}$ for any $V_{\mathrm{G}}$ value. By changing the gate voltage, $V_{\mathrm{T}}$ can be modulated since the alignment condition for resonant tunneling is modified due to a shift in the energy of the molecular band. Inset: 3D plot of the drain-source current as a function of the drain-source voltage $V_{\mathrm{ds}}$ and the gate voltage $V_{\mathrm{g}}$. [Reprinted with permission from G. Maruccio et al., Nano Lett., 2003, 3, 479-483. Copyright 2003 American Chemical Society]

becomes the main challenge of molecular electronics. The selfassembly of single devices at high integration densities using conducting molecular wires could be the key to overcome this challenge and also to go beyond individual components and integrate logic circuits. Proposals already exist to combine demonstrated molecular devices in complex circuits for the fabrication of AND, OR and XOR logic gates, as well as of molecular electronic half- and full-adders. ${ }^{24}$

Recently, logic circuits with field-effect transistors based on single carbon nanotubes ${ }^{56}$ or on assembled nanowire building blocks ${ }^{57}$ have been demonstrated. Researchers at the Delft University of Technology ${ }^{56}$ assembled circuits using an atomic force microscope to select and position nanotubes on top of the gate electrode fabricated by electron beam lithography as well as the source and drain electrodes. The FETs exhibited a transconductance of $0.3 \mu \mathrm{S}$, a very large on/off ratio and a large signal gain (more than $10^{5}$ and 10 , respectively). This technique can be used to assemble different logical elements and the authors reported an inverter, a NOR gate, a static random access memory (SRAM) cell and a ring oscillator using resistor-transistor logic (Fig. 15). In ref. 57, Huang et al. demonstrated OR, AND, NOR logic gates (Fig. 16) using semiconductor nanowires, having the advantage that methods exist for the parallel assembly of nanowires.

The pressing need to realize nanoelectronic circuits by assembling very large numbers of molecular devices capable of performing complex operations has led Y. Chen et al. ${ }^{58}$ to develop nanoscale molecular-switch crossbar circuits where a monolayer of rotaxanes (consisting of two mechanically interlocked compounds) is sandwiched between two mutually perpendicular arrays of nanowires fabricated by nanoinprint lithography. This is a different approach with respect to the logic gate assembled by A. Bachtold et al. which requires AFM imaging to select single walled CNTs (produced by laser ablation) after the electron beam lithography fabrication of $\mathrm{Al}$ gates. This reconfigurable crossbar architecture enables an high throughput, parallel and potentially inexpensive fabrication and interconnection of a large number of nanodevices (molecular switches). Moreover, it can tolerate the presence of defective elements. In such a scheme, each cross point is a molecular reversible, electrically toggled switch which acts as an active memory cell since one bit can be written at each cross point, reversibly switching the resistance of the corresponding devices by selecting its row and column. The authors demonstrated the operation of such circuits as rewritable, non-volatile memories with a density of $6.4{\mathrm{Gbit} \mathrm{cm}^{-2}}^{-2}$ (Fig. 17). Moreover, by setting the resistances at specific cross points, two $4 \times 4$ subarrays of the crossbar were configured to be a nanoscale demultiplexer and multiplexer that were used to read memory bits in a third subarray. 


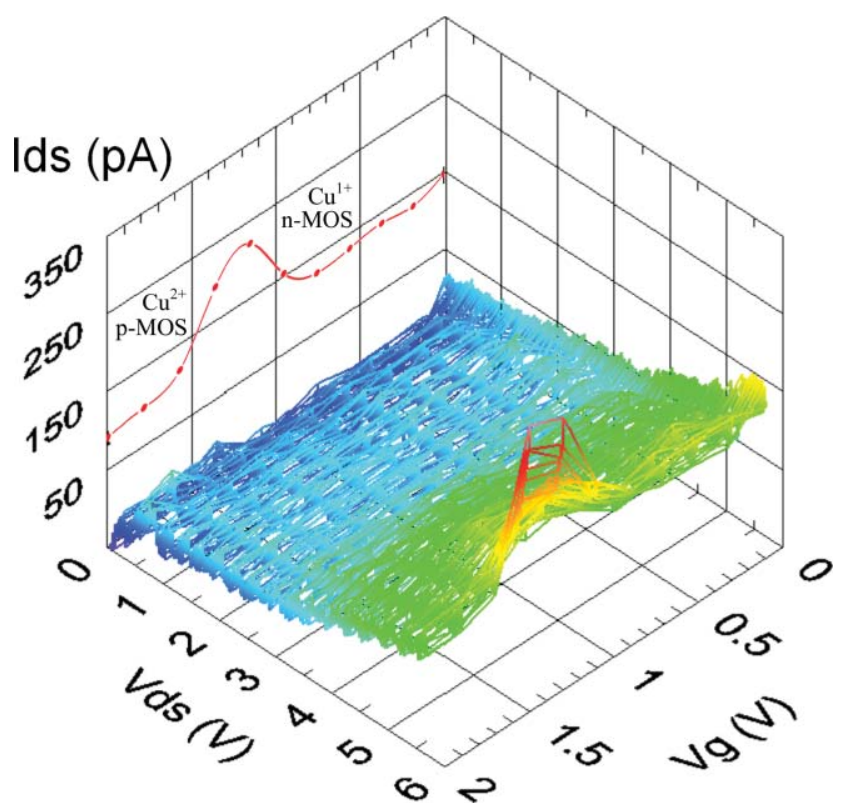

Fig. 14 Characteristics of a protein FET. 3D plot of the drain-source current as a function of the drain-source bias $\left(V_{\mathrm{ds}}\right)$ and of the gate bias $\left(V_{\mathrm{g}}\right)$ measured in the dark and at room temperature. No leakage current was observed to flow between the planar electrodes and the back gate (values as low as a few $\mathrm{pA}$ and a negligible variation with $V_{\mathrm{g}}$ up to $8 \mathrm{~V}$ ). A pronounced resonance centered at $V_{\mathrm{g}}=1.25 \mathrm{~V}$ is present (see also the transfer characteristic at $V_{\mathrm{ds}}=5.5 \mathrm{~V}$ in the projection). In this region, the transconductance changes from positive to negative values. The peak to valley ratio and the FWHM are 2 and $0.3 \mathrm{~V}$, respectively.

\section{Conclusion and outlook}

In conclusion, molecular electronics is a very promising route for future electronic devices. As discussed in section 2, the main milestones for full success of the worldwide effort in this field are the following:

1) two terminal conductance measurement on single molecules (first achieved by scanning probe microscope);

2) demonstration of two terminal molecular devices;

3) demonstration of three terminal molecular devices;

4) integration of the functions at the molecular level;

5) achievement of long-term stability and development of high-throughput fabrication processes.

While the first three milestones have been accomplished (at least at demonstrators level) and molecules acting as transport elements, diodes or transistors have been reported, the latter two requirements represent the next challenge for molecular electronics.

A crucial aspect is to understand and model the coupling of individual molecules to other molecules as well as to macroscopic electrodes, mainly under non-equilibrium (operational) conditions. Note that the connection between the molecules and the electrodes greatly influences the transport characteristics, also determining the device performance. Only the development of design rules and assembly schemes for circuit integration can determine the success of molecular electronics. Moreover, a way to overcome the issues of device ageing, lifetime and reproducibility has to be found in order to transfer molecular electronic devices to mass production at the industrial level.

Finally, some very recent and intriguing results have to be discussed. Until recently, usual electronic devices ignored the spin degree of freedom and relied only on the transport of electrical charges. The emerging field of spintronics (Fig. 18a) exploits the electron spin, instead of its charge, to carry information and create conceptually new spintronic devices with the potential advantages of non-volatility, increased data processing speed, decreased electric power consumption, and increased integration densities compared with standard
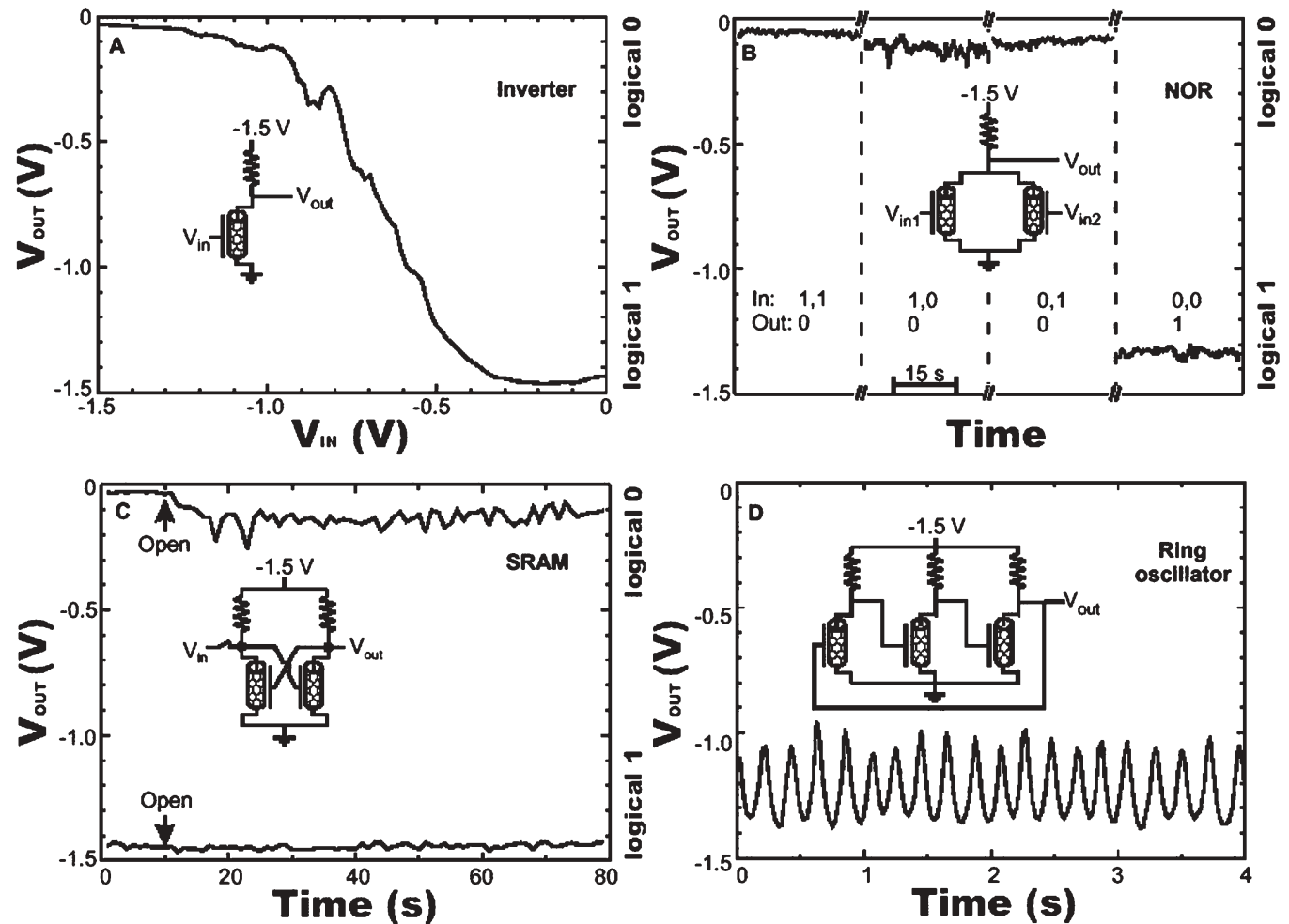

Fig. 15 Nanotube logic circuits using one-, two-, and three-nanotube FETs. (A) $V_{\text {out }}-V_{\text {in }}$ characteristic of a nanotube inverter (NOT gate) fabricated combining a nanotube FET with a resistance. It has only one input. (B) Output voltage of a nanotube NOR gate in the four possible logic address level inputs: $(0,0) ;(0,1) ;(1,0) ;(1,1)$, where logic " 0 " is $0 \mathrm{~V}$ and logic " 1 " is $-1.5 \mathrm{~V}$. This gate has two inputs and its output is "true" (" 1 ") if both inputs are "false" (region 0,0). Otherwise, the output is "false" ("0"). (C) Static random access memory made by two nanotube FETs and two resistances. (D) Output voltage of a nanotube ring oscillator composed of three nanotube FETs. [Reprinted with permission from Bachtold et al., Science, 2001, 294, 1317-1321. Copyright 2001 American Association for the Advancement of Science] 
(a)
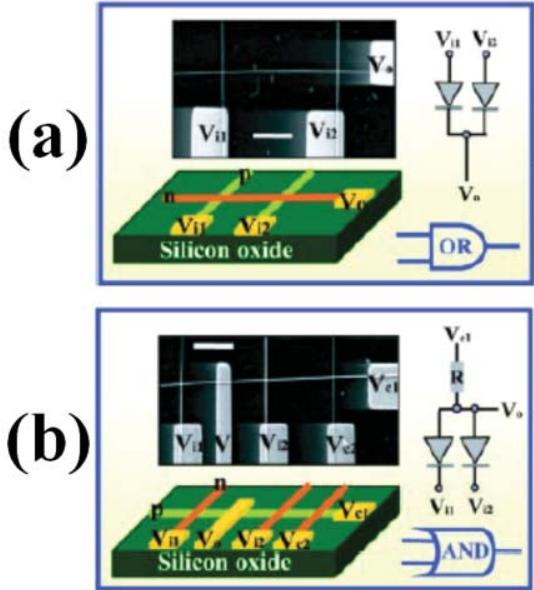

(b)

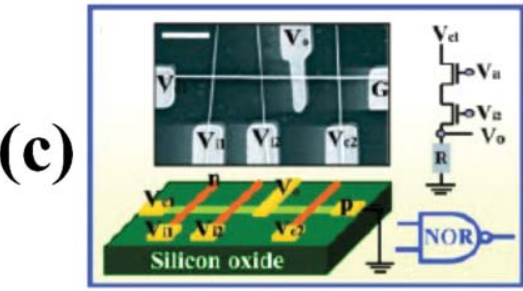

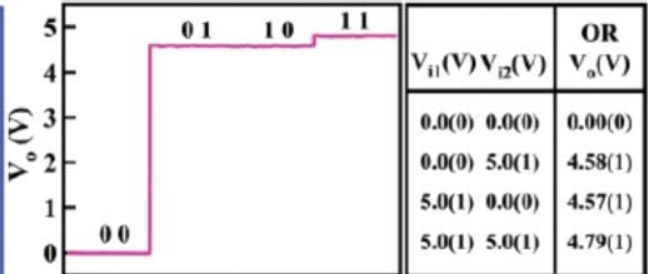

OR Address Level

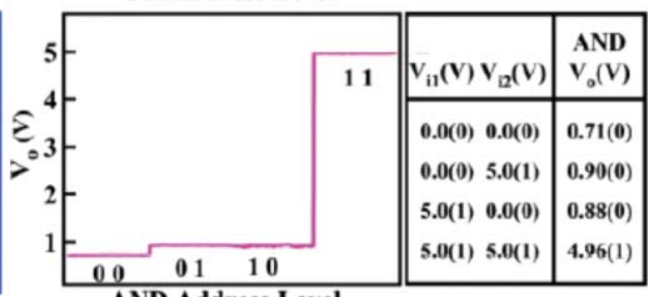

AND Address Level

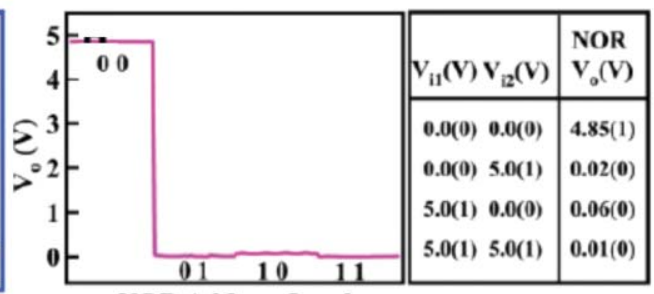

NOR Address Level

Fig. 16 Demonstration of nanowire logic gates, arranged to calculate operation from boolean algebra. (a) Schematics, output voltage and experimental truth table for the OR gate. The logical states " 0 " ("false") and " 1 " ("true") are represented by voltages of $0 \mathrm{~V}$ and $5 \mathrm{~V}$, respectively. (b) AND gate. (c) NOR gate. [Reprinted with permission from Y. Huang et al., Science, 2001, 294, 1313-1316. Copyright 2001 American Association for the Advancement of Science]

semiconductor devices. To successfully incorporate spins, researchers need to efficiently inject, transport, control and detect spin polarization and spin-polarized currents, using new materials if necessary. Taking this challenge, M. Ouyang and D. D. Awschalom ${ }^{59}$ reported coherent spin transfer between molecularly bridged CdSe quantum dots, assembled in an artificial solid by means of dithiol-conjugated molecules. Twocolor time-resolved Faraday rotation (TRFR) measurements have been used to selectively spin inject/detect CdSe quantum dots (QDs) of two different sizes (3.4 and $7.0 \mathrm{~nm}$ ) incorporated in multilayer devices by tuning the pump/probe energy. Even when the probe energy was chosen to inject the large QDs, they

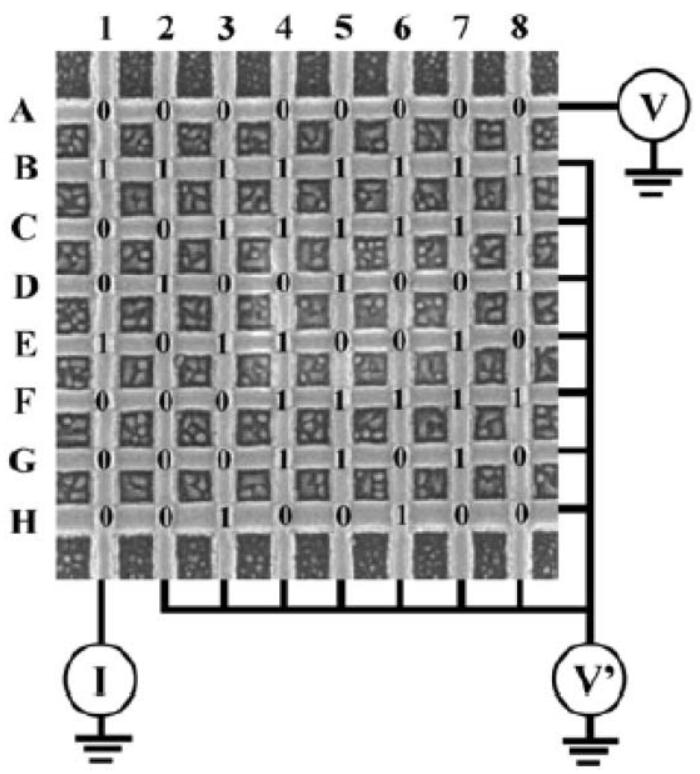

Fig. 17 Crossbar 64-bit random access memory. [Reproduced from Y. Chen et al., Nanotechnology, 2003, 14, 462-468. Copyright 2003 Institute of Physics Publishing] observed two distinct Larmor frequencies, thus attributed to the spin-coherent dynamics of carriers in both types of QDs. These results, suggesting coherent spin transfer from larger to smaller QDs, are very promising for the purpose of developing bottom-up spintronics.

A different approach to molecular-scale computing devices has been proposed by C. Lent et al. ${ }^{60}$ which discussed the possibility of molecular devices operating without the conventional flow of current. While in a standard computer binary information is encoded in the on or off state of a currentwhich can be reversibly switched on or off (using either a gate voltage or a voltage pulse) - the researchers at University of Notre Dame explored a cool computing method based on the concept of quantum-dot cellular automata (QCA, Fig. 18b) extended at the molecular level - where the charge configuration among redox-active molecular sites carries information. An alkane bridge provides the tunneling barrier which couples the states of adjacent dots. The natural logic gate and basis of QCA architecture is the three-input majority gate, where threeinput lines meet at a device cell whose state is determined by the state of the majority of the inputs. It is possible to use it as a programmable AND/OR gate by fixing one of its three inputs: the state of the input $\mathrm{A}$ can be considered as switching the functionality between (B and C) and (B or C). Combined with the inverter, this programmable AND/OR gate provides logical completeness to QCA architecture.

Before concluding, it is right and fair to mention recent results of C. Hettich et al. ${ }^{61}$ which are expected to be useful in the molecular implementation of quantum bits or 'qubits' (Fig. 18c), i.e. in the use of single molecules as supports for qubits. Generally, quantum mechanics is viewed as a drawback of nanoscale systems, being the realm of the potentiality rather than the reality. Thus, dealing with quantum states instead of classical quantities is problematic and the behaviour of particles becomes elusive. However, quantum states are also an unprecedented opportunity to realize a new, very powerful way to computation and quantum computers can solve NP complete problems in polynomial time. In computational 


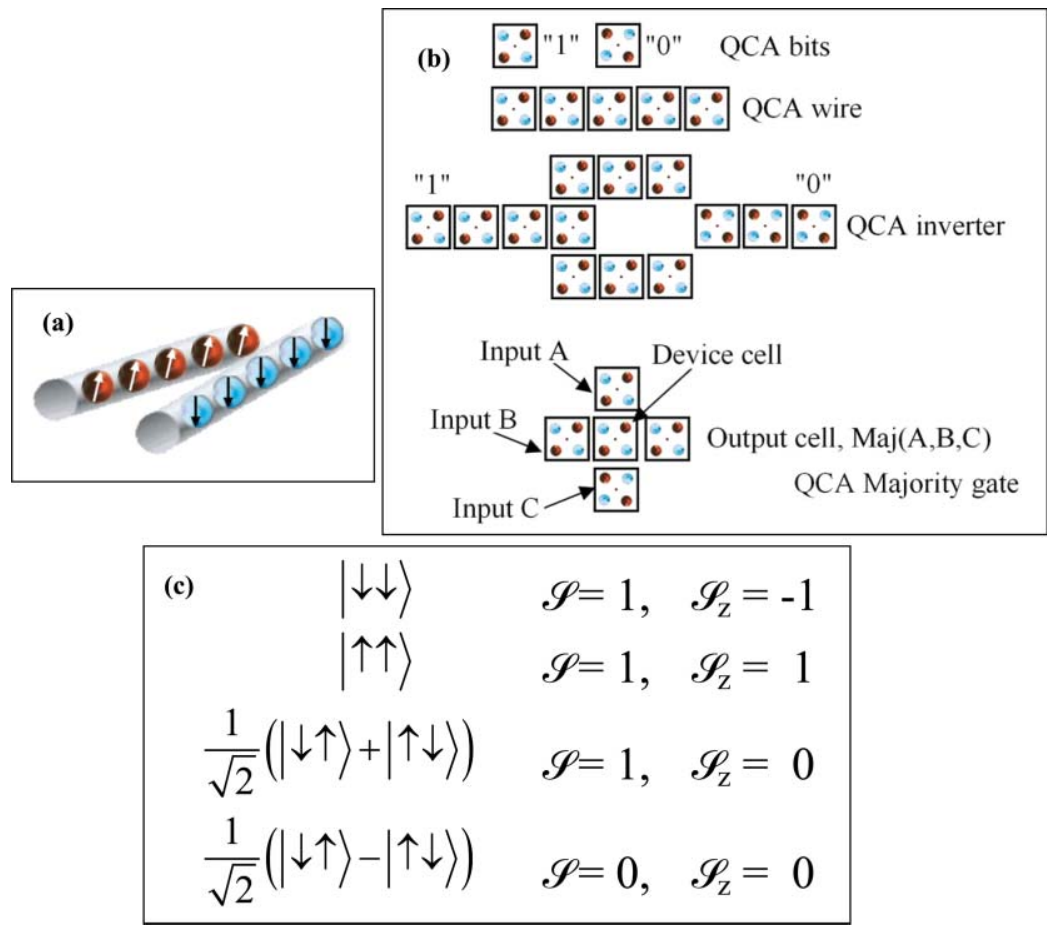

Fig. 18 (a) The concept of spintronics, which exploit the spin degree of freedom to carry information. (b) Quantum cellular automata architecture: bits, wire, inverter and majority gate. (c) Quantum computation exploiting entangled states. A specific example of entangled pairs occurs in the total spin of two electron systems. The two states $S=1, S_{z}=0$ and $S=0, S_{z}=0$ are examples of entangled states.

complexity theory, NP ("non-deterministic polynomial-time") problems are the set of decision problems solvable in polynomial time on a non-deterministic Turing machine. Or, equivalently, YES answers are checkable in polynomial time on a deterministic Turing machine given the right information. The NP-complete problems are the hardest problems in NP. If one could find a way to solve an NP-complete problem quickly, then he could use that algorithm to solve all NP problems quickly. As an example, Shor's algorithm allows one to factorize large whole numbers in polynomial time, with strong implications for the security of much of modern cryptography. C. Hettich et al. resolved two individual fluorescent molecules separated by $12 \mathrm{~nm}$, thanks to the position dependent Stark shift induced by an inhomogeneous electric field. Then, they succeeded in entangling them, realizing a coherent superposition of their excitations, and in demonstrating the feasibility of manipulating the degree of entanglement between molecules. The small decoherence time limits any application, but such an approach presents the advantage that although qubits are physically carried by single molecules, the outcome of a quantum operation can be read optically.

\section{Acknowledgements}

We would like to thank Valentina Arima, Adriana Biasco, Alessandro Bramanti, Franco Calabi, Stefano D'Amico, Eliana D'Amone and Paolo Visconti at NNL-INFM research center in Lecce (Italy). Financial support by NNL-INFM and EC through SAMBA project is gratefully acknowledged.

\section{References}

1 The Internet Technology Roadmap for Semiconductors (ITRS) is available at http://public.itrs.net.

2 P. L. Packan, Pushing the Limits, Science, 1999, 285, 2079-2081.

3 V. Balzani, M. Venturi and A. Credi, in Molecular Devices and Machines - A Journey into the Nano World, Wiley-VCH, 2002.

4 J. M. Lehn, Angew. Chem., Int. Ed. Engl., 1988, 27, 89.

5 J. M. Lehn, Supramolecular Chemistry - concept and perspectives, VCH, Weinheim, 1995.
6 L. L. Looger, M. A. Dwyer, J. J. Smith and H. W. Hellinga, Nature, 2003, 423, 185-190.

7 (a) L. M. Adleman, Science, 1994, 266, 1021; (b) R. S. Braich, N. Chelyapov, C. Johnson, P. W. K. Rothemund and L. Adleman, Science, 2002, 296, 499-502.

8 For example biofuel cells exploiting enzymes that extract energy from compounds (such as glucose) to power life have been reported [T. Chen, S. Calabrese Barton, G. Binyamin, Z. Gao, Y. Zhang, H. Kim and A. Heller, J. Am. Chem. Soc., 2001, 123, $8630-8631$.

9 J. K. Gimzewski, C. Joachim, R. R. Schlittler, V. Langlais, H. Tang and I. Johannsen, Science, 1998, 281, 531-533.

10 N. L. Rosi, J. Eckert, M. Eddaoudi, D. T. Vodak and J. Kim, Science, 2003, 300, 1127-1129.

11 The flow of a liquid on single-walled carbon nanotube bundles induces a voltage in the sample along the direction of the flow [see S. Ghosh, A. K. Sood and N. Kumar, Science, 2003, 299, 10421044.

12 N. N. Lavrik and P. G. Datskos, Appl. Phys. Lett., 2003, 82, $2697-$ 2699.

13 S. S. Wong, J. D. Harper, P. T. Lansbury and C. M. Lieber, J. Am. Chem. Soc., 1998, 120, 603-604.

14 H. Iwai, IEEE J. Solid State Circuits, 1999, 34, 357-366.

15 S. A. Wolf, D. D. Awschalom, R. A. Buhrman, J. M. Daughton, S. von Molnar, M. L. Roukes, A. Y. Chtchelkanova and D. M. Treger, Science, 2001, 294, 1488-1495.

16 (a) A. Steane, Rep. Prog. Phys., 1998, 61, 117-173; (b) F. De Martini, V. Buzek, F. Sciarrino and C. Sias, Nature, 2003, 419, 815-818.

17 A. Aviram and M. A. Ratner, Chem. Phys. Lett., 1974, 29, $277-$ 283.

18 K. Ziemelis, Nature, 1998, 393, 619-620.

19 C. D. Muller, A. Falcou, N. Reckefuss, M. Rojahn, V. Wiederhirn, P. Rudati, H. Frohne, O. Nuyken, H. Becker and K. Meerholz, Nature, 2003, 421, 829-833.

20 B. Crone, A. Dodabalapur, Y. Y. Lin, R. W. Filas, Z. Bao, A. LaDuca, R. Sarpeshkar, H. E. Katz and W. Li, Nature, 2000, 403, 521-524.

21 J. M. Tour, Acc. Chem. Res., 2000, 33, 791-804.

22 S. Cholet, C. Joachim, J. P. Martinez and B. Rousset, Eur. Phys. J. Appl. Phys., 1999, 8, 139-145.

23 Y. V. Kervennic, H. S. J. Van der Zant, A. F. Morpurgo, L. Gurevich and L. P. Kouwenhoven, Appl. Phys. Lett., 2002, 80, 321-323.

24 J. C. Ellenbogen and J. C. Love, Architectures for Molecular Electronic Computers. 1. Logic Structures and an Adder Built 
from Molecular Electronic Diodes MITRE Research Article (The "Pink Book"), available at http://www.mitre.org/tech/nanotech/ Arch_for_MolecElec_Comp_1.html.

25 S. Datta, W. Tian, S. Hong, R. Reifenberger, J. I. Henderson and C. P. Kubiak, Phys. Rev. Lett., 1997, 79, 2530-2533.

26 T. W. Kelley, E. L. Granstrom and C. D. Frisbie, Adv. Mater., 1999, 11, 261-264.

27 R. E. Holmlin, R. Haag, M. L. Chabinyc, R. F. Ismagilov, A. E. Cohen, A. Terfort, M. A. Rampi and G. M. Whitesides, J. Am. Chem. Soc., 2001, 123, 5075-5085.

28 J. Chen, M. A. Reed, A. M. Rawlett and J. M. Tour, Science, 1999, 286, $1550-1552$.

29 M. A. Reed, C. Zhou, C. J. Muller, T. P. Burgin and J. M. Tour, Science, 1997, 278, 252-254

30 C. W. Gwyn, R. Stulen, D. Sweeney and D. Attwood, J. Vac. Sci. Technol. B, 1998, 16, 3142-3149.

31 G. Maruccio, P. Visconti, S. D'Amico, P. Calogiuri, E. D'Amone, R. Cingolani and R. Rinaldi, Microelectron. Eng., 2003, 67-68, 838-844.

32 S. Y. Chou, P. R. Krauss and P. J. Renstrom, Science, 1996, 272, $85-87$.

33 D. Piner, J. Zhu, F. Xu, S. Hong and C. A. Mirkin, Science, 1999, 283, 661-663

34 (a) S. Hong, J. Zhu and C. A. Mirkin, Science, 1999, 286, 523-525; (b) S. Hong and C. A. Mirkin, Science, 2000, 288, 1808-1811.

35 L. M. Demers, D. S. Ginger, S.-J. Park, Z. Li, S.-W. Chung and C. A. Mirkin, Science, 2002, 296, 1836-1838.

36 M. Cavallini, F. Biscarini, S. Leon, F. Zerbetto, G. Bottari and D. A. Leigh, Science, 2003, 299, 531.

37 M. Kölbel, R. W. Tjerkstra, J. Brugger, C. J. M. van Rijn, W. Nijdam, J. Huskens and D. N. Reinhoudt, Nano Lett., 2002, 2 , 1339-1343.

38 M. P. MacDonald, L. Paterson, K. Volke-Sepulveda, J. Arlt, W. Sibbett and K. Dholakia, Science, 2002, 296, 1101-1103.

39 K. Keren, M. Krueger, R. Gilad, G. Ben-Yoseph, U. Sivan and E. Braun, Science, 2002, 297, 72-75.

40 S. Lee, C. Mao, C. E. Flynn and A. M. Belcher, Science, 2002, 296 892-895.

41 R. M. Metzger, B. Chen, U. Holpfner, M. V. Lakshmikantham, D. Vuillaume, T. Kawai, X. Wu, H. Tachibana, T. V. Hughes, H. Sakurai, J. W. Baldwin, C. Hosch, M. P. Cava, L. Brehmer and G. J. Ashwell, J. Am. Chem. Soc., 1997, 119, 10455-10466.

42 J. M. Seminario, A. G. Zacarias and J. M. Tour, J. Am. Chem Soc., 2000, 122, 3015-3020.

43 M. A. Reed, J. Chen, A. M. Rawlett, D. W. Price and J. M. Tour Appl. Phys. Lett., 2001, 78, 3735-3737.
44 S. J. Tans, A. R. M. Verschueren and C. Dekker, Nature, 1998, 393, 49-52.

45 Armchair $(n=m)$ tubes are metallic, while other tubes (chiral and zig zag) are metallic if $n-m=3 l$ where $l$ is an integer, otherwise they are semiconducting with a gap depending only on the diameter [J. W. G. Wildoer, L. C. Venema, A. G. Rinzler, R. E. Smalley and C. Dekker, Nature, 1998, 391, 59-62.

46 J. Appenzeller, J. Knoch, V. Derycke, R. Martel, S. Wind and Ph. Avouris, Phys. Rev.Lett., 2002, 89, 126801

47 H. W. C. Postma, T. Teepen, Z. Yao, M. Grifoni and C. Dekker, Science, 2001, 293, 76-79.

48 J. Park, A. N. Pasupathy, J. I. Goldsmith, C. Chang, Y. Yaish, J. R. Petta, M. Rinkoski, J. P. Sethna, H. D. Abruna, P. L. McEuen and D. C. Ralph, Nature, 2002, 417, 722-725.

49 D. Porath, A. Bezryadin, S. de Vries and C. Dekker, Nature, 2000, 403, 635-638.

50 G. Maruccio, P. Visconti, V. Arima, S. D'Amico, A. Biasco, E. D'Amone, R. Cingolani and R. Rinaldi, Nano Lett., 2003, 3, 479-483.

51 J. Lefebvre, J. F. Lynch, M. Llaguno, M. Radosavljevic and A. T. Johnson, Appl. Phys. Lett., 2002, 75, 3014-3016.

52 G. Maruccio, A. Biasco, S. D'Amico, A. Bramanti, E. D'Amone, P. Visconti, R. Cingolani and R. Rinaldi, Nature Mater., submitted.

53 L. Ke, S. J. Chua, K. Zhang and N. Yakovlev, Appl. Phys. Lett., 2002, 80, 2195-2197.

54 J. O. Lee, G. Lientschnig, F. Wiertz, M. Struijk, R. A. J. Janssen, R. Egberink, D. N. Reinhoudt, P. Hadley and C. Dekker, Nano Lett., 2003, 3, 113-117.

55 C. R. Kagan, A. Afzali, R. Martel, L. M. Gignac, P. M. Solomon, A. G. Schrott and B. Ek, Nano Lett., 2003, 3, 119-124.

56 A. Bachtold, P. Hadley, T. Nakanishi and C. Dekker, Science, 2001, 294, 1317-1320.

57 Y. Huang, X. Duan, Y. Cui, L. J. Lauhon, K. Kim and C. M. Lieber, Science, 2001, 294, 1313-1317.

58 (a) Y. Chen, G. Jung, D. A. A. Ohlberg, X. Li, D. R. Steward, J. O. Jeppesen, K. A. Nielsen, F. Stoddart J and R. S. Williams, Nanotechnology, 2003, 14, 462-468; (b) Y. Chen, D. A. A. Ohlberg, X. Li, D. R. Steward, R. S. Williams, J. O. Jeppesen, K. A. Nielsen, F. Stoddart J, D. L. Olynick and E. Anderson, Appl. Phys. Lett. 2003, 82, 1610-1612.

59 M. Ouyang and D. D. Awschalom, Science, 2003, 301, 1074-1078.

60 C. S. Lent, B. Isaksen and M. Liederman, J. Am. Chem. Soc., 2003 , 125, 1056-1063.

61 C. Hettich, C. Schmitt, J. Zitzmann, S. Kuhn, I. Gerhardt and V. Sandoghdar, Science, 2002, 298, 385-389. 